\title{
Effect of Seasons on Theanine Levels in Different Kenyan Commercially Released Tea Cultivars and Its Variation in Different Parts of the Tea Shoot
}

\author{
Too C. Janet ${ }^{*}$, Wanyoko K. John², Kinyanjui Thomas', Moseti O. Kelvin², \\ Wachira N. Francis ${ }^{3}$ \\ ${ }^{1}$ Department of Chemistry, Egerton University, Njoro, Kenya \\ ${ }^{2}$ Kenya Agricultural and Livestock Research Organization, Tea Research Institute, Kericho, Kenya \\ ${ }^{3}$ Association for Strengthening Agricultural Research in East and Central Africa, Entebbe, Uganda \\ Email: *janettietoo@yahoo.com
}

Received 16 October 2015; accepted 20 November 2015; published 23 November 2015

Copyright (C) 2015 by authors and Scientific Research Publishing Inc.

This work is licensed under the Creative Commons Attribution International License (CC BY).

http://creativecommons.org/licenses/by/4.0/

(c) (i) Open Access

\begin{abstract}
A study was conducted to determine the effect of seasons in $\mathbf{2 3}$ different Kenyan commercially released tea cultivars. This was done for three seasons viz: cold and wet, cold and humid, hot and dry. The cold and wet season was shown to be the best for obtaining high levels of theanine while the hot and dry season resulted in decrease in theanine levels in most of the test cultivars thus showing seasonal variation on the levels of theanine. Theanine content was determined using High Performance Liquid Chromatography (HPLC). On average, TRFK $31 / 8$ contained $1.20 \%$ dw of theanine and highest in green tea while TRFK $56 / 89$ contained $0.97 \% \mathrm{dw}$ of theanine and highest in black tea. Green tea contained higher theanine levels than black tea and this has been shown in most of the test cultivars. To achieve the health benefits of theanine, one should drink 4 cups of tea brewed from cultivars with $0.63 \% \mathrm{dw}$ and more of theanine. Also theanine levels were determined in the different partitions of the tea shoot ( 4 leaves + bud) and the internode contained the highest theanine levels up to $3.26 \% \mathrm{dw}$ for both cultivars compared to the leafy partitions.
\end{abstract}

\section{Keywords}

Cultivars, Partitions, Seasons, Tea, Theanine

${ }^{*}$ Corresponding author.

How to cite this paper: Janet, T.C., John, W.K., Thomas, K., Kelvin, M.O. and Francis, W.N. (2015) Effect of Seasons on Theanine Levels in Different Kenyan Commercially Released Tea Cultivars and Its Variation in Different Parts of the Tea Shoot. Food and Nutrition Sciences, 6, 1450-1459. http://dx.doi.org/10.4236/fns.2015.615149 


\section{Introduction}

Tea is one of the most popular drinks in the world and has been perceived as being healthy. Tea drinks have been reported to have chemopreventive effects that inactivate potentially harmful free radical oxygen in the body system [1]-[5]. Moreover, tea drinks are known to prevent obesity [6], breast cancer [7], coronary heart [8] and cardiovascular [9] diseases. These beneficial effects have been attributed to the presence of compounds such as polyphenols, amino acids, vitamins, carbohydrates, and purine alkaloids [10]. Theanine is a non-protein amino acid that was first discovered in tea leaves [11] and has also been found in bay bolete mushroom, Xerocomus badius [12] [13].

Theanine is a predominant amino acid, $1 \%-2 \%$ of the dry weight of the leaves comprises about $50 \%$ of total protein [14]. It was first identified by Sakato in tea leaves as the main free amino acid component that can influence the major umami constituents of tea [15]. Various health effects have been associated with L-theanine, such as neuroprotective effects, which ultimately enhanced nervous attention. On the basis of two parallel techniques, i.e. electroencephalographic (EEG) and behavioral modules indicated that L-theanine influences functionality of brain [16]. Current consensus regarding theanine has explained its effect in mitigation of mental and physical stress due to its ability to cross the blood-brain barrier [17]. Moreover, it enhances cognition and behavior in a systematic manner with caffeine. In addition, L-theanine helps boost immunity against infection by enhancing the disease-fighting ability of gamma delta $\mathrm{T}$ cells [18]. Recently, array of biochemical modulations proved theanine's effectiveness to enhance the activity of several antitumor agents, including doxorubicin (DOX) [19]. In addition to neuro-functionality and antitumor activities, its role as hepatic protective agent is also well documented. During oxidative stress it protects the liver damage by increasing glutathione level via metabolism through glutaminase and c-GTP [20].

Theanine levels in different tea cultivars have been determined in other tea growing countries especially in China, Japan but no much work has been done on Kenyan released tea cultivars. This study was carried out to determine the effect of seasons on theanine levels in different selected released cultivars and in different parts of the tea shoot. Since no much work has been done on this, it has not yet been known if the Kenyan cultivars are of health benefit with respect to this amino acid.

\section{Materials and Method}

\subsection{Experimental Site and Design}

The experimental design was a completely randomized design. This study was carried out at the Timbilil estate $\left(0^{\circ} 22^{\prime}\right.$ South, $35^{\circ} 21^{\prime}$ East, elevation 2180 m a.m.s.l.) located at Tea Research Institute (TRI) based in Kericho, Kenya. The determination of the theanine levels in the selected commercially released cultivars was done for three seasons, where the first season (July to September, 2011) was done when weather conditions were cold and wet, the second season (October to December, 2011) was cold and humid (less rainfall) and the third season (January to March, 2012) which was hot and dry. Two different leaf colored cultivars were also partitioned into five partitions and theanine levels were determined in these different partitions.

\subsection{Sample Collection and Pre-Treatment}

Twenty three (23) cultivars were selected for this experiment, viz., TRFK 31/8, TRFK 12/12, TRFK 31/11, TRFK 301/4, TRFK 301/5, TRFK K-Purple, TRFK 6/8, TRFK 303/577, TRFK 303/216, TRFK 303/178, TRFK 303/259, TRFK 7/9, TRFK 7/3, TRFK 7/14, TRFK 54/40, TRFK 56/89, TRFK 11/26, TRFK 100/5, TRFK 108/82, TRFCA SFS 150, TRFK 11/52, TRFK 12/19, TRFK 11/4. The samples were taken to the institute's miniature factory and processed as green and black tea from the respective cultivar.

TRFK 301/5 (ordinary green leaf colored cultivar) and TRFK 91/1 (purple leaf colored cultivar) were also sampled in order to determine their theanine levels in different parts of the tea shoot (4 leaves + a bud). The samples were taken to the institute's miniature factory and steamed on arrival. They were then partitioned into first leaf, second leaf, third leaf, fourth leaf and internode.

\subsection{Sample Preparation}

$1.00 \pm 0.0001 \mathrm{~g}$ of a finely ground sample was weighed into a $200 \mathrm{ml}$ beaker and $100 \mathrm{ml}$ of boiling double dis- 
tilled water was added. The sample was then allowed to brew for 5 minutes on a hot plate (MHK-4, Mrc, Germany) while stirring. The sample was allowed to cool, made up to volume by double distilled water and filtered using a $0.45 \mu \mathrm{m}$ membrane into sample vials. Theanine is only soluble in water and insoluble in organic solvents like ethanol, methanol, chloroform and ether [21] [22].

\subsection{Preparation of Working Solutions}

To obtain a standard stock solution, $50.0 \pm 0.01 \mathrm{mg}$ of pure L-theanine was accurately weighed into a $50 \mathrm{ml}$ volumetric flask and dissolved with double distilled water by the aid of sonication using Ultra Sonic Bath (XB14, Grant, England) and made up to the volume with the double distilled water. Standard working solutions were prepared by serial dilution of the standard stock solution and were in the concentration range of $20-80 \mu \mathrm{g} / \mathrm{ml}$.

\subsection{Chromatographic Determination of Theanine}

The theanine content determination in the test samples was done by High Performance Liquid Chromatography (HPLC). The HPLC used was a Shimadzu LC 20 fitted with an SIL 20A auto sampler and an SPD-20 UV-Visible detector (set at $210 \mathrm{~nm}$ ) with a class LC10 chromatograph workstation, manufactured in Kyoto, Japan. The method was developed by [23], has been undergoing international ring test and described by [24].

\subsection{Statistical Analysis of Data}

The results obtained were subjected to Analysis of Variance (ANOVA) using MSTAT statistical software for windows version 2.10 at $p<0.05$. The Least Significant Difference (LSD) test was used for mean separation where statistically significant differences were observed. Data are presented as a mean of triplicate determinations \pm standard deviation (SD).

\section{Results and Discussion}

Theanine levels were determined using HPLC and a sample of a chromatogram obtained from theanine standard and test cultivar have been shown in Figure 1 and Figure 2 respectively. Green and black tea were processed from the respective cultivars and the results obtained were as follows.

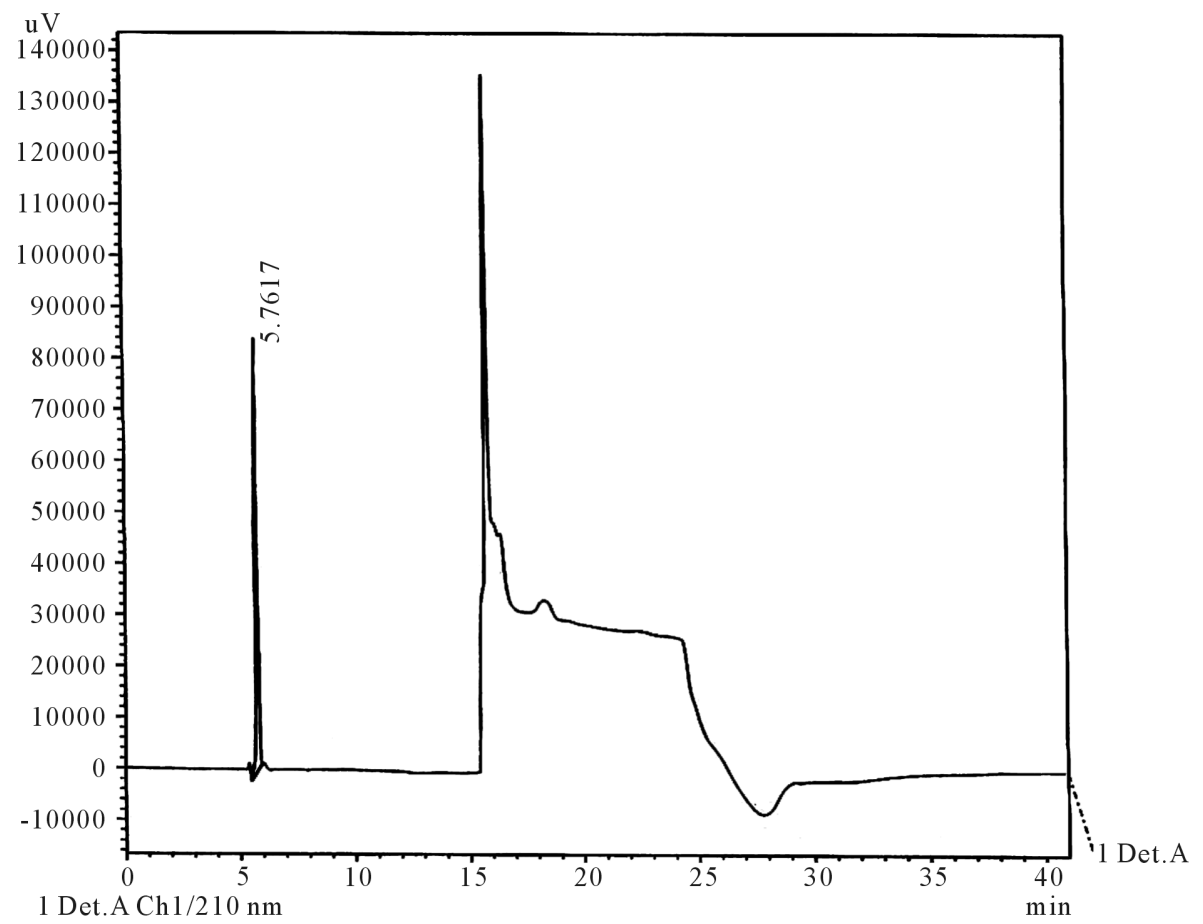

Figure 1. HPLC chromatogram for a theanine standard. 


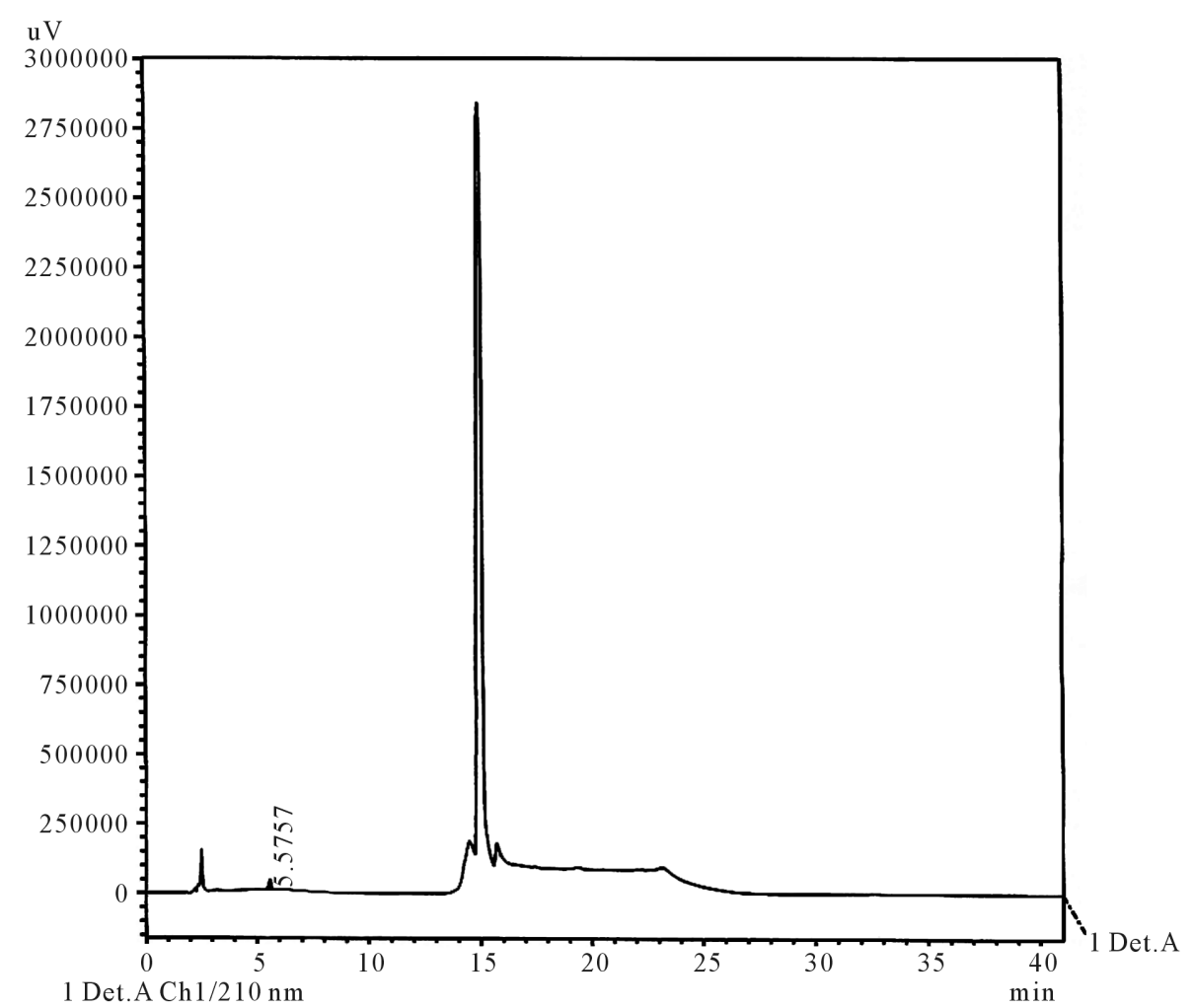

Figure 2. HPLC chromatogram obtained for one of the test cultivar.

\subsection{Green Tea}

The levels of theanine in the 23 cultivars of green tea processed during the three seasons were as graphically represented in Figure 3. During the first season (July-September, 2011), the linear calibration curve obtained ( $y$ $\left.=8707.4 \mathrm{x}+6075.4, r^{2}=0.9996\right)$, demonstrated adequate linearity and was used to calculate theanine levels during this season for both green and black tea. Theanine levels in the twenty three cultivars ranged between $0.54 \%-1.50 \% \mathrm{dw}$. TRFCA SFS 150 contained the highest amount of theanine while TRFK 6/8 contained the lowest amount during this season. TRFK 31/8 and TRFK 7/9 theanine levels were not significantly different from each other $(p>0.05)$. Most of the cultivars analyzed contained $0.6 \% \mathrm{dw}$ of theanine and more. TRFK K-Purple was the only purple cultivar in this study and it was among the cultivars with high theanine levels. TRFK K-Purple contained theanine in comparable amounts to TRFK 7/3 and TRFK 11/26. Therefore it is double rich as it contains both anthocyanins (potent antioxidants) and theanine.

During the second season (October-December, 2011), the same number of cultivars were analyzed and a linear calibration line, $y=10284 x+2520.9\left(r^{2}=0.9999\right)$ was obtained and used to calculate theanine levels for both green and black tea. The amount of theanine in green tea ranged between $0.59 \%-1.34 \%$ dw. The cultivar that contained the highest theanine level in green tea was TRFCA SFS 150 with 1.34\% dw and the lowest was TRFK $7 / 14$ with $0.59 \% \mathrm{dw}$. On average, most of the green tea contained $0.7 \% \mathrm{dw}$ of theanine. Though TRFCA SFS 150 contained highest theanine levels $(1.34 \% \mathrm{dw})$, its levels were not significantly different $(p>0.05)$ from that contained in TRFK 56/89 (1.28\% dw) and TRFK 7/9 (1.28\% dw). TRFK 7/14 theanine levels of 0.59\% dw were not significantly different $(p>0.05)$ from the levels in TRFK 301/4 (0.63\% dw), TRFK 303/178 $(0.64 \%$ $\mathrm{dw})$, TRFK K-Purple (0.73\% dw), and TRFK 31/11 (0.69\% dw), even though it contained the least amount of theanine after subjecting the results to Least Significant Difference (LSD) test. TRFK K-Purple contained 0.73\% dw of theanine and its content was not significantly different $(p>0.05)$ from TRFK 303/577 among other cultivars.

In the third season (January-March, 2011), the same number of cultivars were analyzed and a linear calibration line, $y=10100 \mathrm{x}-1209.1\left(r^{2}=0.9961\right)$ was obtained which was then used to calculate theanine levels for both green and black teas. The theanine levels in green tea ranged between $0.14 \%-1.09 \%$ dw. The cultivar that 


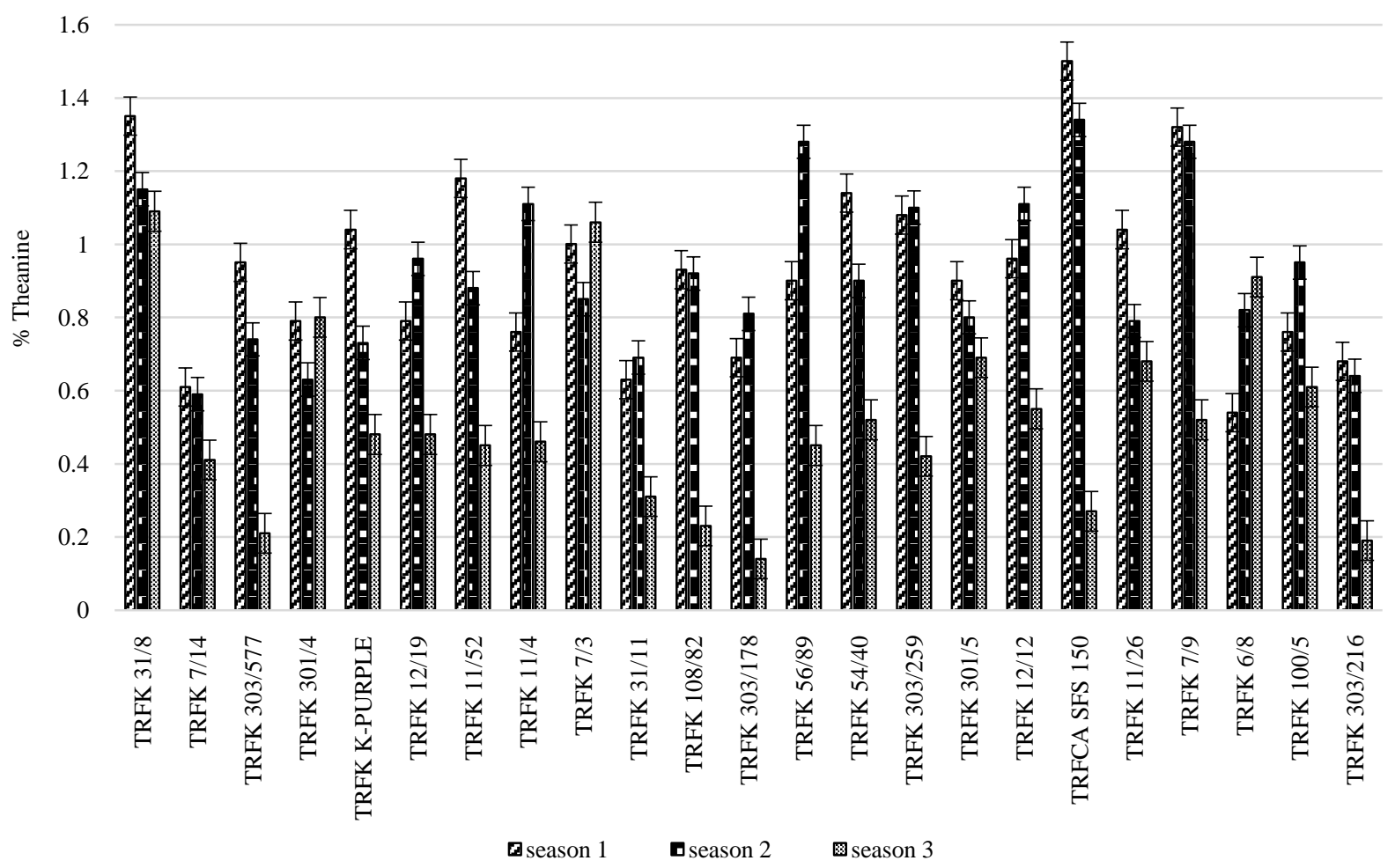

Figure 3. Theanine levels in green tea processed from 23 Kenyan commercially released cultivars during three seasons.

contained the highest amount of theanine was TRFK 31/8 with 1.09\% dw and the lowest was TRFK 303/178 which contained $0.14 \% \mathrm{dw}$. The theanine level in TRFK $31 / 8$ was not significantly different $(p>0.05)$ from the content in TRFK 7/3 (1.06\% dw) though when the means were ranked TRFK 7/3 was the second highest. TRFK K-Purple contained $0.48 \% \mathrm{dw}$ of theanine and this was not significantly different $(p>0.05)$ from the theanine levels in TRFK 12/19 (0.48\% dw), TRFK 54/40 (0.52\% dw) and TRFK 7/9 (0.52\% dw) which were green leaf colored cultivars.

Theanine levels varied during the three seasons with the first and second season having high levels of theanine. The third season showed a decrease in theanine levels in most of the cultivars except TRFK 7/3, TRFK 301/4 and TRFK 6/8 whose theanine levels were highest during this season. TRFK 301/4 is a drought resistant clone while TRFK 7/3 is slightly resistant to drought [25] and this could be the reason why theanine levels in these clones were high. TRFK 6/8 is not drought resistant but contained high levels of theanine. In a study conducted by [24], theanine levels were shown to be affected by sunlight in that it decreases its levels since it is a biosynthetic precursor of catechins. There could be genetic traits responsible for these results which need to be further studied. There was a variation in theanine levels among the analyzed cultivars in all the seasons. Every cultivar has different genetic composition and some traits might be responsible for this variation and research has to be done on this. The best season to obtain optimum theanine levels was the first and second season when the climatic conditions were favourable.

\subsection{Black Tea}

The results for black tea processed from the 23 cultivars of tea for the three seasons were as graphically represented in Figure 4. Black tea theanine levels range was between $0.42 \%-0.98 \%$ dw during season one. The cultivar that contained the highest level of theanine was TRFK 108/82 with $0.98 \%$ dw and the lowest was TRFCA SFS 150 with $0.42 \%$ dw. Most of the black teas contained on average $0.5 \%$ dw of theanine. TRFK KPurple contained $0.80 \% \mathrm{dw}$ and was among cultivars with the highest levels of theanine with its levels being comparable to ordinary green leaf colored cultivars: TRFK 31/8 (0.83\% dw), TRFK $301 / 5$ (0.85\% dw) and TRFK $11 / 52(0.88 \%$ dw). Nearly half of the cultivars analyzed contained theanine below that of TRFK K-Purple. 


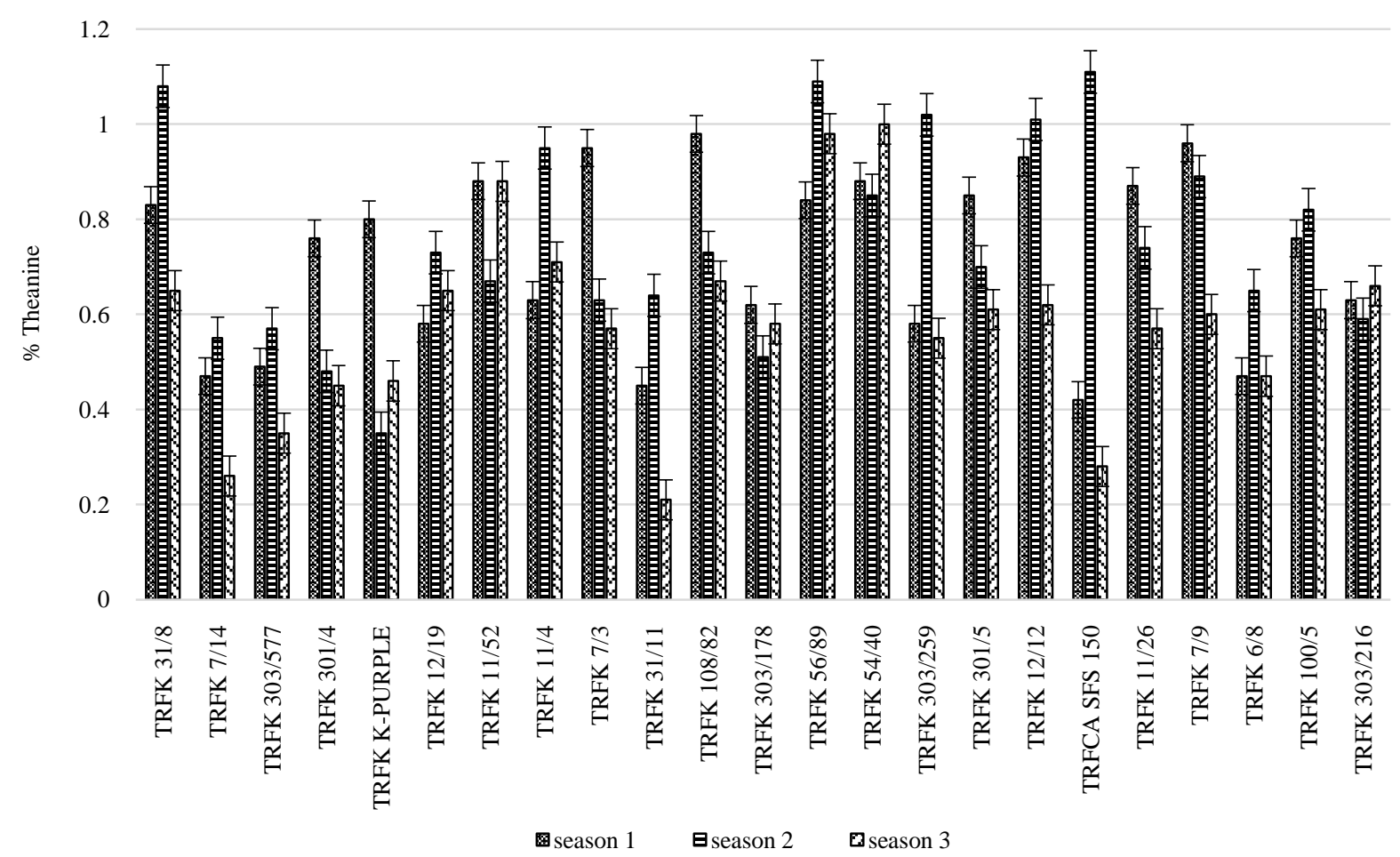

Figure 4. Theanine levels in black tea processed from 23 Kenyan commercially released tea cultivars during three seasons.

The best quality black tea cultivar TRFK $6 / 8$ contained $0.47 \% \mathrm{dw}$ and this was not significantly different ( $p>$ $0.05)$ from its progeny TRFK 303/577 $(0.49 \% \mathrm{dw})$ but significantly different $(p<0.05)$ from TRFK 303/178 $(0.62 \% \mathrm{dw})$ and TRFK $303 / 216(0.63 \% \mathrm{dw})$ which are also its progenies.

The levels of theanine ranged between $0.35 \%-1.11 \% \mathrm{dw}$ during season two. The cultivar that contained the highest amount of theanine was TRFCA SFS 150 and TRFK K-Purple with $0.35 \% \mathrm{dw}$ contained the lowest. The theanine levels in TRFCA SFS 150 was not significantly different $(p>0.05)$ from the levels in TRFK 12/12, TRFK 56/89, TRFK 31/8 and TRFK 303/259. Most of the cultivars contained $0.6 \% \mathrm{dw}$ of theanine on average during this season. The best quality black tea cultivar TRFK $6 / 8$ contained $0.65 \% \mathrm{dw}$ and was not significantly different $(p>0.05)$ from TRFK 301/5 $(0.70 \% \mathrm{dw})$, TRFK 7/3 $(0.63 \% \mathrm{dw})$, TRFK $31 / 11(0.64 \% \mathrm{dw})$ TRFK $11 / 52(0.67 \% \mathrm{dw})$ and TRFK 303/216 (0.59\% dw) among others. TRFK 303/259 being a progeny of TRFK 6/8 contained $1.02 \% \mathrm{dw}$ of theanine which was higher and significantly different $(p<0.05)$ from its mother bush (parent). TRFK 303/178, a progeny of TRFK $6 / 8$ contained $0.51 \% \mathrm{dw}$ of theanine which was lower and significantly different $(p<0.05)$ from TRFK 6/8 (parent). The differences in theanine levels between the TRFK 6/8 with its progenies could be due to inherited genes from the mother bush. Some genetic traits could be more in one progeny and less in another. This is yet to be established by plant breeders.

The theanine levels ranged between $0.21 \%-1.00 \% \mathrm{dw}$ during the third season. The cultivar that contained the highest level of theanine was TRFK 54/40 with $1.0 \% \mathrm{dw}$ and the lowest was TRFK $31 / 11$ with $0.21 \% \mathrm{dw}$. TRFK 31/11 is affected severely by drought and this could be the reason its theanine levels were low. Theanine levels in TRFK 54/40 were not significantly different $(p>0.05)$ from the levels in TRFK 56/89 $(0.98 \% \mathrm{dw})$ and TRFK $11 / 52(0.88 \% \mathrm{dw})$. TRFK K-purple cultivar contained $0.46 \% \mathrm{dw}$ and was also in the range of some ordinary green leaf colored cultivars. TRFK K-Purple theanine levels were not significantly different $(p>0.05)$ from the levels in TRFK $6 / 8(0.47 \% \mathrm{dw})$ which is well known for the production of high quality black tea. Theanine levels in TRFK 6/8 $(0.47 \% \mathrm{dw})$ was also not significantly different $(p>0.05)$ from two of its progenies: TRFK 303/178 $(0.58 \% \mathrm{dw})$ and TRFK 303/259 $(0.55 \% \mathrm{dw})$ but significantly different $(p<0.05)$ from TRFK $303 / 216(0.66 \% \mathrm{dw})$ which is also its progeny. This could also be due to genetic traits inherited from the mother bush.

Theanine levels varied in the three different seasons for the different cultivars. Some clones contained high theanine levels during season one then decreased during season two while others increased also during the 
second season. In the third season, theanine levels decreased in most of the cultivars except a few which increased. This could have been due to the climatic conditions [26] [27] during this season which was hot and dry. There is no much documented work that has been done on these cultivars seasonally. Theanine level variation in cultivars could also be due to enzymes responsible for theanine synthesis: glutamine synthetase, glutamine synthase, glutamate dehydrogenase, alanine transaminase alanine decarboxylase and theanine hydrolase which could be differentially expressed in different clones [28].

\subsection{A Comparison of the Theanine Levels in Green and Black Teas during the Three Seasons}

The results from three different seasons were also averaged and graphically represented as shown in Figure 5. The results from the three seasons showed that green tea contained more theanine than black tea in most of the cultivars analyzed. TRFK 31/8 contained the highest theanine level in green tea with $1.20 \% \mathrm{dw}$ and the lowest being TRFK $303 / 216$ with $0.50 \%$ dw while in black tea TRFK 56/89 contained the highest theanine with $0.97 \%$ $\mathrm{dw}$ and TRFK 7/14, TRFK 31/11 contained the lowest with 0.43\% dw each. TRFK K-Purple cultivar contained theanine comparable to the ordinary green leaf colored cultivars such as TRFK 301/4, TRFK 301/5, TRFK 12/19, TRFK 11/4, TRFK 6/8, and TRFK 100/5 in green tea and even higher than some other green leaf colored cultivar in this study. TRFK $6 / 8$ contained $0.53 \% \mathrm{dw}$ and has all along been known to be best for black tea manufacture. TRFK 6/8 levels were lower than those of its progenies.

Most of the green tea processed and analyzed in this study contained more theanine than black tea processed from the same cultivars. The delay in processing might have also resulted in other cultivars having no significant difference $(p>0.05)$ in both black and green tea like TRFK K-Purple and TRFK 7/3 during the three seasons. Theanine levels variations in cultivar could have been due to difference in genetic traits, since every cultivar has different gene traits. TRFK 6/8 being well known for black tea, contained theanine in comparable amounts to other cultivars sampled in this study and especially K-Purple which is also anthocyanin rich. A study conducted by [29] analyzed some cultivars that were done in this study though from a different location (Kangaita) for

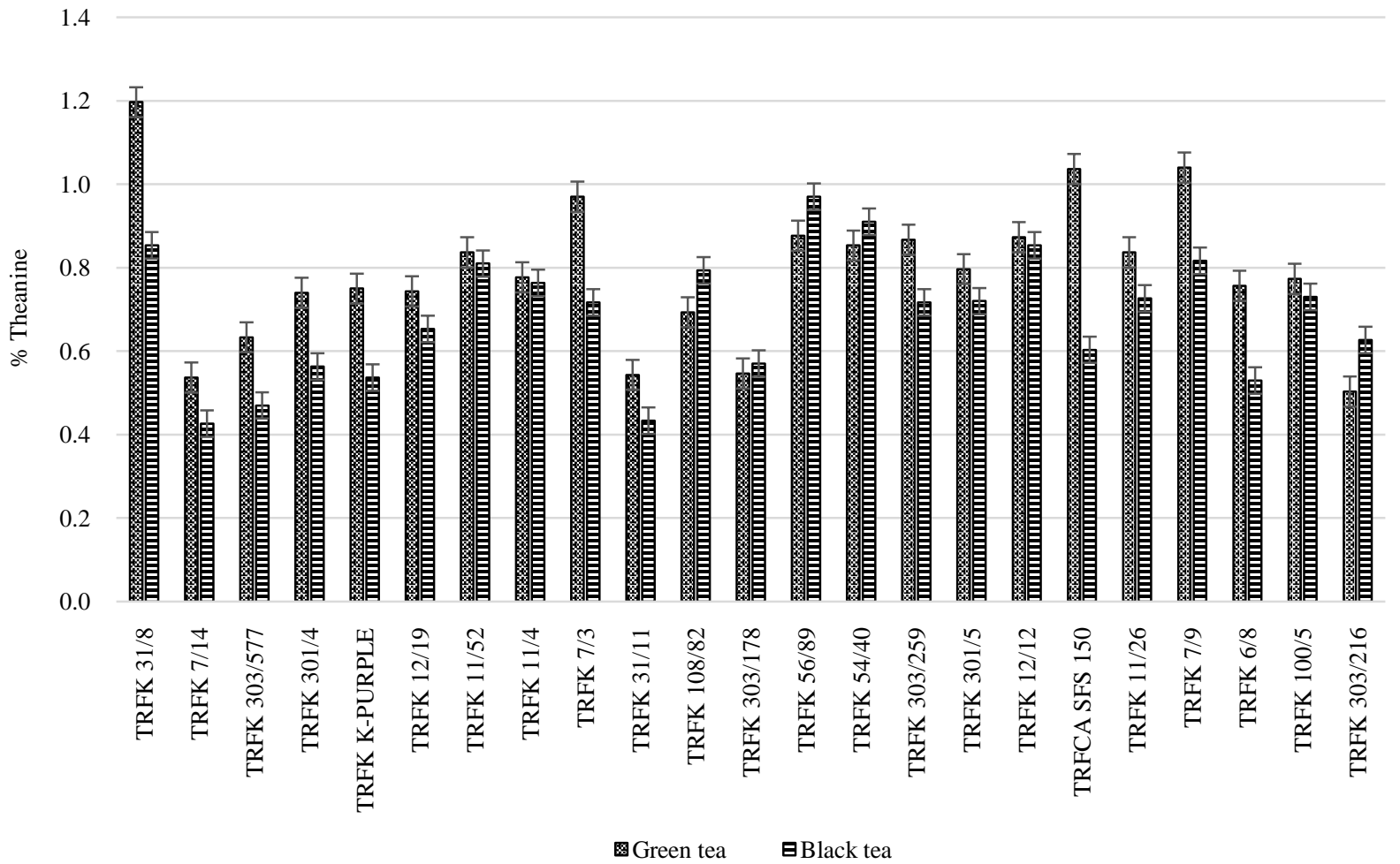

Figure 5. Average levels of theanine for green and black teas processed from 23 Kenyan commercially released tea cultivars during three seasons. 
theanine levels in green tea and itwas shown that different growing conditions affect the levels of this amino acid. TRFK 31/8, TRFK 303/216, TRFK 6/8, TRFK 303/577 and K-Purple contained 1.01\%, 1.26\%, 0.92\%, 1.0\%, $1.10 \%$ in [29] study and $1.20 \%, 0.63 \%, 0.76 \%, 0.63 \%, 0.75 \%$ respectively in this study. Further, this suggests regional variations, an aspect not investigated in the current study. Indeed, climatic and/or regional variations and agronomic practices have been reported to influence the accumulation of theanine in tea leaves [26] [27].

Theanine has been reported to be antihypertensive and this was shown to be dose dependent. A dose of 50 $200 \mathrm{mg}$ per day was shown to be of health benefit. In this study, most of the green and black tea processed from their respective cultivars were shown to be of health benefit since their theanine levels on average were high. For every cup of tea, 2 g of tea leaves (tea bags) are used and upon conversion into the amount contained in a cup, a cultivar like TRFK 31/8 (1.20\% dw) contained $24 \mathrm{mg}$ of theanine. After drinking $3 \mathrm{cups}$ (72 mg) of tea will be of health benefit, being antihypertensive among others. The lowest theanine levels on average obtained in this study was $0.43 \% \mathrm{dw}$ in TRFK 31/11, TRFK 7/14 and both were black tea. One would take 6 cups (51.6 $\mathrm{mg}$ ) of tea to achieve the health benefits of theanine from these two cultivars.

\section{Difference in Leaf Parts and Coloration}

Theanine levels in the different partitions of the tea shoot varied in the two cultivars (Figure 6): TRFK $301 / 5$ and TRFK 91/1. TRFK 91/1 contained 0.50\% dw while TRFK 301/5 contained 0.79\% dw of theanine in the bud. TRFK $91 / 1$ contained $0.22 \%$ dw while TRFK 301/5 contained $0.40 \%$ dw of theanine in the first leaf. TRFK $91 / 1$ contained $0.33 \% \mathrm{dw}$ while TRFK $301 / 5$ contained $0.70 \%$ dw of theanine in the second leaf. TRFK 91/1 contained $0.34 \% \mathrm{dw}$ while TRFK $301 / 5$ contained $0.74 \%$ dw of theanine in the third leaf. TRFK $91 / 1$ contained $0.42 \%$ dw while TRFK 301/5 contained $0.59 \%$ dw of theanine in the fourth leaf. TRFK 91/1 contained 0.94\% dw while TRFK $301 / 5$ contained $3.26 \%$ dw of theanine in the internode. Theanine levels in these different partition for the two clones were significantly different $(p<0.05)$.

TRFK 91/1 was a purple leaf colored cultivar while TRFK 301/5 was an ordinary green leaf colored cultivar. TRFK 301/5 contained the highest levels of theanine in all the partitions and had an average of $1.08 \%$ dw while TRFK $91 / 1$ had $0.46 \%$ dw. The internode was the only partition that contained the highest amount of theanine in all the partitions for the two clones. The results in this study showed that green leaf colored cultivar contained more theanine than purple leaf coloured cultivar. A study conducted by [29], showed that purple leaf colored

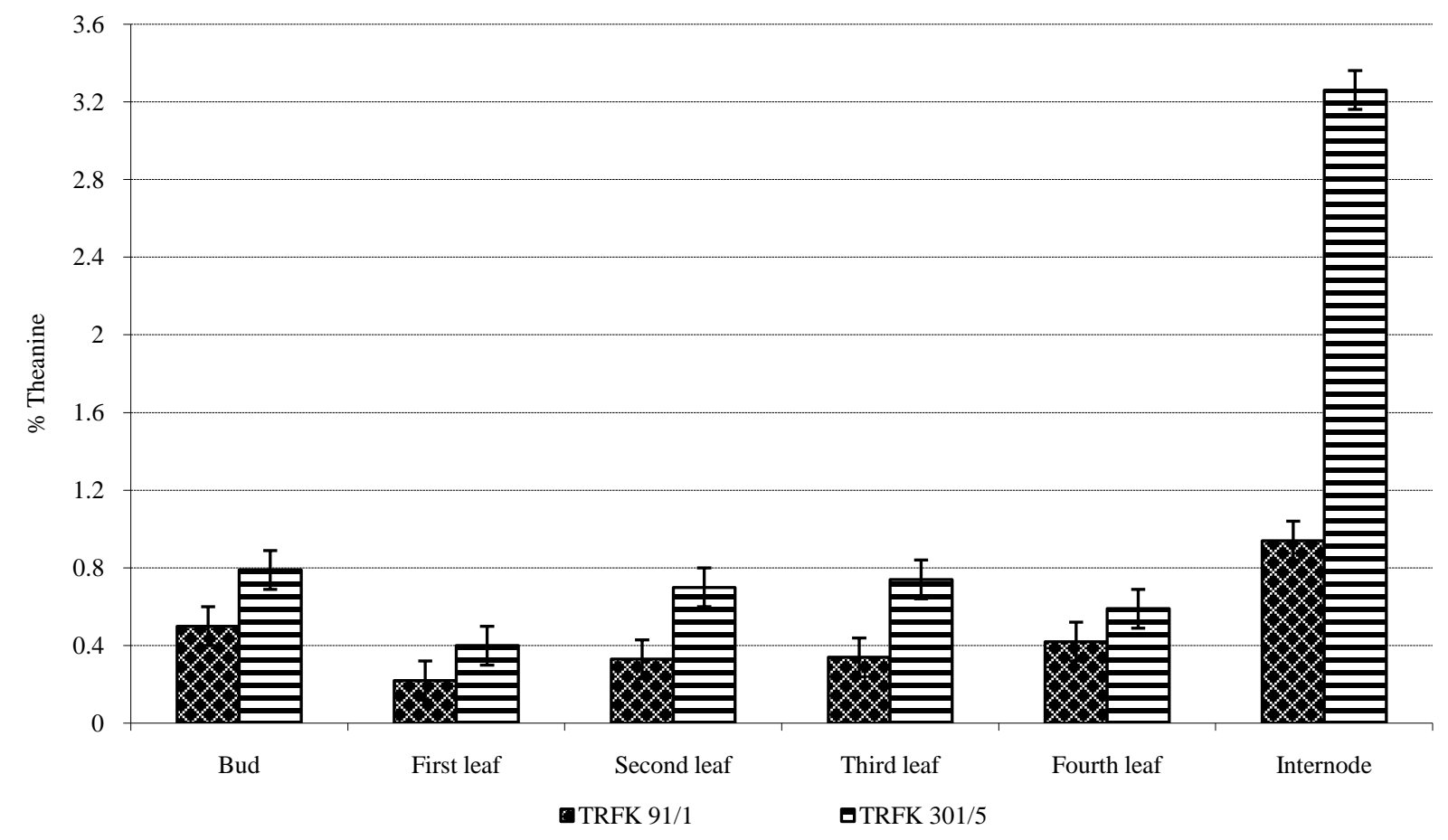

Figure 6. Theanine levels in the different leaf colored cultivar. 
cultivars $(1.44 \% \mathrm{dw})$ contained more theanine than green leaf colored cultivars $(1.12 \% \mathrm{dw})$ and this was not in agreement with this study. This could have been due to difference in the climatic conditions [26] [27] [30] [31] of the locations where these two studies were conducted: Kericho and Kangaita.

\section{Conclusion}

Theanine levels varied with different test cultivars. The levels were also affected by different climatic conditions (seasons) with the hot and dry season showing a decrease in theanine levels in most of the clones. Green tea contained higher theanine levels than black tea. The ordinary green leaf colored cultivar contained higher theanine levels than the purple leaf colored cultivar which was also anthocyanin rich. The internode was shown to contain high levels of theanine compared to the leafy partitions.

\section{Acknowledgements}

This work has been published with permission from the Institute Director, Tea Research Institute.

\section{References}

[1] Andlauer, W. and Héritier, J. (2011) Rapid Electrochemical Screening of Antioxidant Capacity (RESAC) of Selected Tea Samples. Food Chemistry, 125, 1517-1520. http://dx.doi.org/10.1016/j.foodchem.2010.10.046

[2] Chen, C., Yu, R., Owuor, E.D. and Kong, A.N.T. (2000) Activation of Antioxidant-Response Element (ARE), Mitogen-Activated Protein Kinases (MAPKs) and Caspases by Major Green Tea Polyphenol Components during Cell Survival and Death. Archives of Pharmacal Research, 23, 605-612. http://dx.doi.org/10.1007/bf02975249

[3] Higdon, J.V. and Frei, B. (2003) Tea Catechins and Polyphenols: Health Effects, Metabolism, and Antioxidant Functions. Critical Reviews in Food Science and Nutrition, 43, 89-143. http://dx.doi.org/10.1080/10408690390826464

[4] Leung, L.K., Su, Y., Chen, R., Zhang, Z., Huang, Y. and Chen, Z.Y. (2001) Theaflavins in Black Tea and Catechins in Green Tea Are Equally Effective Antioxidants. Journal of Nutrition, 131, 2248-2251.

[5] Stewart, A.J., Mullen, W. and Crozier, A. (2005) On-Line High Performance Liquid Chromatography Analysis of the Antioxidant Activity of Phenolic Compounds in Green and Black Tea. Molecular Nutrition \& Food Research, 49, 52-60. http://dx.doi.org/10.1002/mnfr.200400064

[6] Maki, K.C., Reeves, M.S., Farmer, M., Yasunaga, K., Matsuo, N., Katsuragi, Y., Komikado, M., Tokimitsu, I., Wilder, D., Jones, F., Blumberg, J.B. and Cartwright, Y. (2009) Green Tea Catechin Consumption Enhances Exercise-Induced Abdominal Fat Loss in Overweight and Obese Adults. Journal of Nutrition, 139, 264-270. http://dx.doi.org/10.3945/jn.108.098293

[7] Shrubsole, M.J., Lu, W., Chen, Z., Shu, X.O., Zheng, Y., Dai, Q., Gu, K., Ruan, Z.X., Gao, Y.T. and Zheng, W. (2009) Drinking Green Tea Modestly Reduces Breast Cancer Risk. Journal of Nutrition, 139, 310-316. http://dx.doi.org/10.3945/jn.108.098699

[8] Higdon, G.E.J., Ruxton, C.H.S. and Leed, A.R. (2007) Black Tea-Helpful or Harmful? A Review of the Evidence. European Journal of Clinical Nutrition, 61, 3-18. http://dx.doi.org/10.1038/sj.ejcn.1602489

[9] Duffy, S.J., Keaney Jr., J.F., Holbrook, M., Gokce, N., Swerdloff, P.L., Frei, B. and Vita, J.A. (2001) Short- and LongTerm Black Tea Consumption Reverses Endothelial Dysfunction in Patients with Coronary Artery Disease. Circulation. Journal of the American Heart Association, 104, 151-156. http://dx.doi.org/10.1161/01.cir.104.2.151

[10] Bolling, B.W. and Chen, C.Y (2009) Tea and Health: Preventive and Therapeutic Usefulness in the Elderly. Current Opinion in Clinical Nutrition \& Metabolic Care, 12, 42-48. http://dx.doi.org/10.1097/MCO.0b013e32831b9c48

[11] Sakato, Y. (1949) The Chemical Constituents of Tea: A New Amide Theanine. Journal of Agricultural and Food Chemistry, 23, 262-267.

[12] Syu, K.Y., Lin, C.L., Huang, H.C. and Lin, J.K. (2008) Determination of Theanine, GABA, and Other Amino Acids in Green, Oolong, Black, and Puerh Teas with Dabsylation, and High Performance Liquid Chromatography. Journal of Agricultural and Food Chemistry, 56, 7637-7643. http://dx.doi.org/10.1021/jf801795m

[13] Deng, W.W., Shinjiro, O. and Hiroshi, A. (2010) Distribution and Biosynthesis of Theanine in Theaceae Plants. Plant Physiology and Biochemistry, 47, 70-72.

[14] Palva, S. and Palva J.M. (2007) New Vistas for Alpha-Frequency Band Oscillations. Trends in Neurosciences, 30, 150158. http://dx.doi.org/10.1016/j.tins.2007.02.001

[15] Hideyuki, S., Shunsuke, I., Nobukazu, M. and Hidehiko, K. (2002) Enzymatic Production of Theanine, an "Umami” Component of Tea, from Glutamine and Ethylamine with Bacterial $\gamma$-Glutamyl Trans Peptidase. Enzyme and Microbial 
Technology, 31, 884-889.

[16] DeMejia, E.G., Ramirez-Mares, M.V. and Puangpraphant, S. (2009) Bioactive Components of Tea: Cancer, Inflammation and Behavior. Brain, Behavior, and Immunity, 23, 721-731. http://dx.doi.org/10.1016/j.foodchem.2010.10.046

[17] Boris, K., Nir, G., Amos, D. and Korczyn, H. (2009) Smoking and Tea Consumption Delay Onset of Parkinson’s Disease. Parkinsonism and Related Disorders, 15, 41-46. http://dx.doi.org/10.1016/j.foodchem.2010.10.046

[18] Sugiyama, T. and Sadzuka, Y. (2003) Theanine and Glutamate Transporter Inhibitors Enhance the Antitumor Efficacy of Chemotherapeutic Agents. Biochimica et Biophysica Acta, 5, 47-59. http://dx.doi.org/10.1016/s0304-419x(03)00031-3

[19] Popkin, B.M. (2010) Recent Dynamics Suggest Selected Countries Catching up to US Obesity. American Journal of Clinical Nutrition, 91, 1284S-1288S. http://dx.doi.org/10.3945/ajcn.2009.28473C

[20] Van der Pijl, P.C., Chen, L. and Mulder, T.P.J. (2010) Human Disposition of L-Theanine in Tea or Aqueous Solution. Journal of Functional Foods, 2, 239-244. http://dx.doi.org/10.1016/j.jff.2010.08.001

[21] Wan, X., Zhang, Z. and Li, D. (2009) Chemistry and Biological Properties of Theanine. In: Ho, C.T., Lin, J.K. and Shahidi, F., Eds., Tea and Tea Products, CRC Press, Boca Raton, 255-274.

[22] Chu, D.C. and Juneja, L.R. (1997) General Chemical Composition of Green Tea and Its Infusion. In: Yamamoto, T., Juneja, L.R., Chu, D.C. and Kim, M., Eds., Chemistry and Applications of Green Tea, CRC Press, Boca Raton, 13-22.

[23] Engelhardt, U.H. and Simonides, M. (2007) Schnellmethode zur Bestimmung von Theanin in Tee. Lebensmittelchemie (im Druck).

[24] Too, J.C., Kinyanjui, T., Wanyoko, J.K. and Wachira, F.N. (2015) Effect of Sunlight Exposure and Different Withering Durations on Theanine Levels in Tea (Camellia sinensis). Food and Nutrition Sciences, 6, 1014-1021. http://dx.doi.org/10.4236/fns.2015.611105

[25] Wachira, F.N., Kamunya, S.M., Chalo, R., Maritim, T. and Kinyangi, T. (2012) TRFK Clonal Catalogue. Tea Research Foundation of Kenya (TRFK), 15-140.

[26] Chen, H.Y., Lin-Shiau, S.Y. and Lin, J.K. (2009) Pu-erh Tea: Its Manufacturing and Health Benefits. In: Ho, C.T., Lin, J.K. and Shahidi, F., Eds., Tea and Tea Products: Chemistry and Health-Promoting Properties, CRC Press, Boca Raton, 9-15.

[27] Chen, Y.L., Jiang, Y.M., Duan, J., Shi, J., Xue, S. and Kakuda, Y. (2010) Variation in Catechin Contents in Relation to Quality of "Huang Zhi Xiang” Oolong Tea (Camellia sinensis) at Various Growing Altitudes and Seasons. Food Chemistry, 119, 648-652. http://dx.doi.org/10.1016/j.foodchem.2009.07.014

[28] Li, Q., Huang, J., Liu, S., Li, J., Yang, X., Liu, Y. and Liu, Z. (2011) Proteomic Analysis of Young Leaves at Three Developmental Stages in an Albino Tea Cultivar. Proteome Science, 9, 44-49. http://dx.doi.org/10.1186/1477-5956-9-44

[29] Kilel, E.C., Faraj, A.K., Wanyoko, J.K., Wachira, F.N. and Mwingirwa, V. (2013) Green Tea from Purple Leaf Coloured Tea Clones in Kenya-Their Quality Characteristics. Journal of Food Chemistry, 141, 769-775. http://dx.doi.org/10.1016/j.foodchem.2013.03.051

[30] Lin, J.K., Lin, C.L., Liang, Y.C., Lin-Shiau, S.Y. and Juan, I.M. (1998) Survey of Catechins, GA, and Methylxanthines in Green, Oolong, Pu-erh and Black Teas. Journal of Agricultural and Food Chemistry, 46, 3635-3642. http://dx.doi.org/10.1021/jf980223x

[31] Jeng, K.C., Chen, C.S., Fang, Y.P., Hou, R.C.W. and Chen Y.S. (2007) Effect of Microbial Fermentation on Content of Statin, GABA, and Polyphenols in Pu-erh Tea. Journal of Agricultural and Food Chemistry, 55, 8787-8792. http://dx.doi.org/10.1021/jf071629p 